\title{
Panax ginseng has no effect on indices of glucose regulation following acute or chronic ingestion in healthy volunteers
}

\author{
Jonathon L. Reay ${ }^{1}{ }^{*}$, Andrew B. Scholey ${ }^{2}$, Anthea Milne ${ }^{1}$, J. Fenwick ${ }^{3}$ and David O. Kennedy ${ }^{1}$ \\ ${ }^{1}$ Brain Performance and Nutrition Research Centre, Northumbria University, Newcastle upon Tyne NE1 8ST, UK \\ ${ }^{2}$ NICM Centre for Study of Natural Medicines and Neurocognition, Brain Science Institute, Swinburne University, \\ Melbourne VIC 3122, Australia \\ ${ }^{3}$ Clinical Biochemistry, Gateshead Health NHS Foundation Trust, Gateshead NE9 6SX, UK \\ (Received 28 March 2008 - Revised 10 September 2008 - Accepted 12 September 2008 - First published online 19 November 2008)
}

In the absence of effective pharmacotherapy for diabetes there has been an increase in the use of, and research into, alternative treatment strategies These include exercise, dietary interventions and the use of supplements including extracts of ginseng. Two separate, placebo-controlled, doubleblind, cross-over studies investigating the effects of chronic ingestion of Panax ginseng (study 1 used G115, study 2 used Cheong Kwan Jang) on glycated $\mathrm{Hb}$ (HbA1c; study 1, $n$ 18; study 2, $n$ 11), fasting plasma insulin (study 1, $n$ 17; study 2, $n$ 12), fasting plasma glucose and postprandial response (following breakfast) (study $1, n 23$; study $2, n 14$ ) in healthy volunteers are reported. In both studies it was found that Panax ginseng had no effect on any gluco-regulatory parameter investigated. These results are not consistent with those reported for a diabetic sample (albeit using slightly different outcomes). These results would suggest that chronic use of Panax ginseng by non-diabetic individuals will have little long-term effect on glucose regulation. The benefits to glucose regulation associated with long-term ginseng use may only be present in populations with compromised glucose control; however, further research is needed to confirm such a speculation.

Panax ginseng: Glucose response: Insulin: Glycated Hb levels

The increasing incidence of diabetes ${ }^{(1)}$ is a global concern, especially when the available treatment appears to be insufficient to halt the disease progression and the majority of type 2 diabetes patients eventually require insulin therapy ${ }^{(2)}$. It is estimated that diabetes is likely to be the fifth leading cause of death globally ${ }^{(3)}$. These considerations have led to an increase in the use of, and research into, alternative treatment strategies, including exercise and dietary interventions. The latter include herbal supplements including extracts of ginseng.

The term 'ginseng' usually refers to the dried root of several species in the plant genus Panax (Araliaceae family). The two most common species are Panax ginseng (Asian ginseng) and Panax quinquefolius (American ginseng), both of which have documented acute hypoglycaemic effects ${ }^{(4-15)}$. The acute hypoglycaemic effects of Panax quinquefolius have been demonstrated in a series of randomised, placebo-controlled studies in diabetic and healthy human subjects. Compared with placebo, reductions in the blood glucose response to a $25 \mathrm{~g}$ (and $75 \mathrm{~g}^{(15)}$ ) glucose challenge have been reported during a $120 \mathrm{~min}$ oral glucose tolerance test in both diabetic patients who had ingested 3,6 and $9 \mathrm{~g}^{(9,10)}$, and healthy participants administered 1, 2, 3 and $9 \mathrm{~g}$ Panax quinquefolius ${ }^{(9,11,15)}$. However, Sievenpiper et al. ${ }^{(16)}$ failed to report any hypoglycaemic effect of $6 \mathrm{~g}$ Panax quinquefolius, administered $40 \mathrm{~min}$ before, or concurrently with, a $75 \mathrm{~g}$ glucose drink in twelve healthy volunteers. Additionally, Sievenpiper et al. ${ }^{(17)}$ reported that there was no effect of eight other widely used ginseng types (Sanchi, Siberian, American, Asian, Korean red, Japanese, wild American and Vietnamese) on indices of glycaemic control following a $75 \mathrm{~g}$ oral glucose tolerance test in healthy volunteers. However, an extract of Panax ginseng was associated with increased blood glucose levels and insulin response following the glucose load ${ }^{(17)}$. With regards to Panax ginseng, a further two studies have reported significant reductions in fasting blood glucose levels measured $1 \mathrm{~h}$ after a single dose of 200 and $400 \mathrm{mg}$ in healthy volunteers but no effect on postprandial glucose (following a $25 \mathrm{~g}$ glucose drink) response ${ }^{(13,14)}$. While there are discrepancies between the findings of the acute studies, these may be attributable to differences in populations (healthy and diabetic), dietary states (fasting and postprandial) and ginseng types (Asian and American). Additionally, treatment doses, timing and the level of glucose loads have differed between studies. Nevertheless there is a growing consensus that both Panax ginseng and Panax quinquefolius are capable of acute glycaemic modulation in both healthy and diabetic populations.

In line with the acute effects briefly delineated earlier, ginseng may also be promising as a potential chronic treatment

Abbreviation: $\mathrm{HbA1c}$, glycated $\mathrm{Hb}$.

* Corresponding author: Dr Jonathon L. Reay, fax +44 191 2273190, email j.reay@unn.ac.uk 
in diabetes. A reduction in fasting blood glucose levels and glycated $\mathrm{Hb}$ (HbA1c; an index of blood glucose over the past month or so) were reported following 8 weeks of administration of 100 and $200 \mathrm{mg} / \mathrm{d}$ of an unspecified ginseng extract in eighteen participants with type 2 diabetes mellitus ${ }^{(18)}$. Similarly, Tetsutani et al. ${ }^{(8)}$ reported that 24 months of treatment with $3-4.5 \mathrm{~g} / \mathrm{d}$ Korean red Panax ginseng decreased HbAlc in thirty-four type 2 diabetics compared with controls. With regards to Panax quinquefolius, a decrease in fasted blood glucose and $\mathrm{HbA} 1 \mathrm{c}$ has been reported in twenty-four type 2 diabetic patients following 8 weeks of administration of $1 \mathrm{~g}$ of a proprietary ginseng extract, taken $40 \mathrm{~min}$ before each meal ${ }^{(10)}$. Similarly, Vuksan et al. ${ }^{(12)}$ reported improved glucose regulation following chronic supplementation with Panax ginseng. Nineteen well-controlled type 2 diabetes patients received Panax ginseng for 12 weeks. Panax ginseng ( $2 \mathrm{~g}$ ) was ingested $40 \mathrm{~min}$ before each of three daily meals. Between-groups comparisons were made on eleven indices of glucose regulation, presented under three categories: (1) HbA1c; (2) fasting indices (fasting plasma glucose, fasting plasma insulin and homeostasis model assessment (HOMA)); and (3) postprandial response to a $75 \mathrm{~g}$ oral glucose tolerance test (mean plasma glucose, mean plasma insulin, peak plasma glucose, peak plasma insulin, plasma glucose area under the curve, plasma insulin area under the curve and oral glucose tolerance test-insulin sensitivity index). Results indicate that following 12 weeks of Panax ginseng supplementation there was no difference in HbAlc, fasting plasma glucose or peak plasma glucose. All other indices of glucose regulation that were measured were improved in the treatment group.

As with the series of acute studies it is of clinical interest to investigate the glycaemic effects following chronic ingestion of ginseng, in a healthy non-diabetic population. Additionally, given that no study to date has investigated the acute glycaemic effects beyond the clinically relevant $2 \mathrm{~h}$ post-dose measurement point, it is of interest to investigate ginseng's glycaemic effects beyond this point. Specifically, to investigate whether glucose levels continue to fall, beyond normoglycaemic levels, once they have returned to baseline levels.

The present paper reports, for the first time, the results from two double-blind, placebo-controlled, balanced, cross-over studies. These studies assessed the effects of 4 and 8 weeks of ingestion of two Panax ginseng extracts on indices of glycaemic regulation $(\mathrm{HbA} 1 \mathrm{c}$, fasting plasma insulin, fasting plasma glucose) and at $3 \mathrm{~h}$ post-breakfast, in healthy volunteers.

\section{Participants and methods}

\section{Participants}

Both study 1 and study 2 were approved by the Northumbria University Division of Psychology Ethics committee and conducted in accordance with the Declaration of Helsinki. Prior to participation each participant gave informed consent and completed a medical health questionnaire. All participants reported that they were in good health (free from heart disorders, respiratory disorders, high blood pressure, epilepsy, panic attacks and diabetes) and were not taking any illicit social drugs. Additionally, they were free from 'over-the-counter' or prescribed medications, with the exception, for some female volunteers, of the contraceptive pill. Heavy smokers (more than ten cigarettes per day) were excluded from both studies. All participants fell within the normal healthy BMI range (participants who fell outside the normative range were excluded from the study). All participants fasted overnight and were alcohol- and caffeine-free for $12 \mathrm{~h}$ prior to, and during, assessment days. In both studies volunteers were paid $£ 150$ for participation. No participant took part in both studies.

Study 1. Thirteen female and twelve male volunteers, recruited through local media advertisements, participated in study 1. Due to a number of missing data points (blood could not be collected or finger prick analysis reported an error) the dataset was reduced to twenty-three (twelve males, eleven females; mean age 35.6 (SD 10.8) years) for finger prick blood glucose levels; reduced to eighteen (ten males, eight females; mean age 33.3 (SD 10.4) years) for $\mathrm{HbA} 1 \mathrm{c}$ and further reduced to seventeen (eight males, nine females; mean age 32.8 (SD 9.7) years) for fasting plasma insulin. Treatment compliance was assessed through verbal communication: participants were asked to record any days that they forgot to take their dose. Two participants reported forgetting to take their dose when in the placebo condition. A further two participants reported that they forgot to take their dose when in the active treatment condition. One participant forgot to take day 14's treatment dose while the second forgot to take the treatment on day 6. All participants were blind to the treatment condition throughout the study and all were included in the analysis.

Study 2. Thirteen female and five male volunteers, recruited through local media advertisements, participated in study 2. Due to a number of missing data points (blood could not be collected or finger prick analysis reported an error) the dataset was reduced to fourteen (five males, nine females; mean age 38.4 (SD 10.6) years) for finger prick blood glucose levels; reduced to twelve (four males, eight females; mean age 40.75 (SD 9.64) years) for fasting plasma insulin and further reduced to eleven (three males, eight femles; mean age 41.5 (SD 9.7) years) for HbA1c. Treatment compliance was assessed through verbal communication: participants were asked to record any days that they forgot to take their dose. One participant reported a failure to take one day's dose when in the active treatment condition (day 17 of the treatment regimen) due to unforeseen working commitments. Participants were blind to their treatment condition throughout the study and all participants were included in the analysis.

\section{Physiological measures}

Glycated $H b$. A $2.5 \mathrm{ml}$ venous blood sample was taken from the arm of each participant at the beginning of each testing day (days 1, 29, 57). Blood samples were collected in a monovette containing EDTA and whole blood was stored at $-20^{\circ} \mathrm{C}$ prior to analysis. HbA1c levels were established using a Tosoh G7 HbAlc analyser. This system uses a cation exchange column to separate $\mathrm{Hb}$ components by different ionic charge. The various components of $\mathrm{Hb}$, including A1c, are separated into six fractions and assayed.

Fasting plasma insulin. A $2.5 \mathrm{ml}$ blood sample was taken from the arm of each participant at the beginning of each testing day (days 1, 29, 57). Blood samples were collected in a serum gel monovette and immediately centrifuged for 
$10 \mathrm{~min}$ at $3000 \mathrm{rpm}$ at $7^{\circ} \mathrm{C}$ using an Allegra $\mathrm{X}-22 \mathrm{R}$ centrifuge supplied by Beckman Coulter. The resulting supernatant was poured off into an Eppendorf tube and kept at $-20^{\circ} \mathrm{C}$ prior to analysis. Blood insulin levels were established using a solid-phase enzyme-amplified sensitivity immunoassay (BioSource INS-EASIA) performed on microtitre plates. The assay uses monoclonal antibodies directed against distinct epitopes of insulin.

Capillary finger prick blood samples. Capillary finger prick blood glucose was sampled pre-dose and $3 \mathrm{~h}$ post-dose on days 1, 29 and 57. Blood glucose levels were measured using a Reflotron Plus diagnostic machine and Reflotron test sticks, $\mathrm{CV}$ is $1.8 \%$ in the normal range and $2.5 \%$ in the normal range for repeatability (within-series imprecision) and reproducibility (day-to-day imprecision), respectively (Roche Diagnostics, Germany). The reliability of the test has previously been confirmed ${ }^{(19)}$.

Treatments. Active treatments and placebo capsules (study 1, containing soyabean oil, partly hydrogenated soyabean oil, yellow beeswax, lecithin, yellow iron oxide; study 2 , information is not available), matched for size, colour, opacity and odour were provided by the manufacturers. Prior to the commencement of each study, a disinterested third party, who had no other involvement in the study, prepared the treatments for each individual participant (treatment order was counterbalanced and participants were randomly allocated to a treatment regimen) and sealed them in containers marked only with the participant code and study day numbers (129 ; 30-57). Each participant ingested two capsules every day for $57 \mathrm{~d}$ (on days 1, 29 and 57 treatments were ingested in the laboratory). The individual capsules contained either an inert placebo or $100 \mathrm{mg}$ Panax ginseng extract G115 in study 1 (Pharmaton SA, Lugano, Switzerland) or $100 \mathrm{mg}$ Panax ginseng extract in study 2 (Cheong Kwan Jang, Korea Ginseng Corporation, Seoul, Korea). Depending on the condition to which the participant was allocated, the combination of capsules treatments corresponded to a dose of
$0 \mathrm{mg}$ (placebo) or $200 \mathrm{mg}$ G115 (study 1) or $200 \mathrm{mg}$ Korean ginseng (study 2).

\section{Procedure}

In both study 1 and study 2 participants received each treatment (placebo and $200 \mathrm{mg}$ Panax ginseng) for $57 \mathrm{~d}$ in total, with an intervening wash-out period of $27 \mathrm{~d}$ between treatments when all participants received placebo. Treatment order was counterbalanced across participants and they were randomly allocated to a treatment regimen. Capillary finger prick blood and venous whole blood $(2 \times 2.5 \mathrm{ml})$ was sampled on each study day (days $1,29,57)$, in both treatment regimens (placebo and $200 \mathrm{mg}$ ), in study 1 and study 2, immediately prior to the ingestion of that day's dose and then again $3 \mathrm{~h}$ post-dose (finger prick sample only). Before leaving the laboratory on each study day, participants were provided with a container containing treatments for each day (either days $2-28$ or days $30-56$ ) until the next laboratory visit. Treatments on days 1, 29 and 57 of each period were consumed in the laboratory. All participants were in an overnight fasted state during pre-dose blood sampling and each participant consumed an identical breakfast on each of their study days, immediately after treatment ingestion (see Fig. 1). Typical light breakfasts consisted of toast, cereal or fruit (selfselected by the participant) and varied between participants.

\section{Statistics}

All data were analysed using the Minitab statistical package version 13.1 (Minitab Ltd, Coventry, UK). Treatment effect on HbA1c and plasma insulin were investigated by two-way repeated measures ANOVA (treatment (placebo, $200 \mathrm{mg}) \times$ day $(1,29570))$ to reveal any chronic effects. Treatment effects on capillary finger prick blood glucose levels were investigated by three-way repeated measures ANOVA (treatment (placebo, 200 mg) $\times$ day $(1,29,57) \times$ session

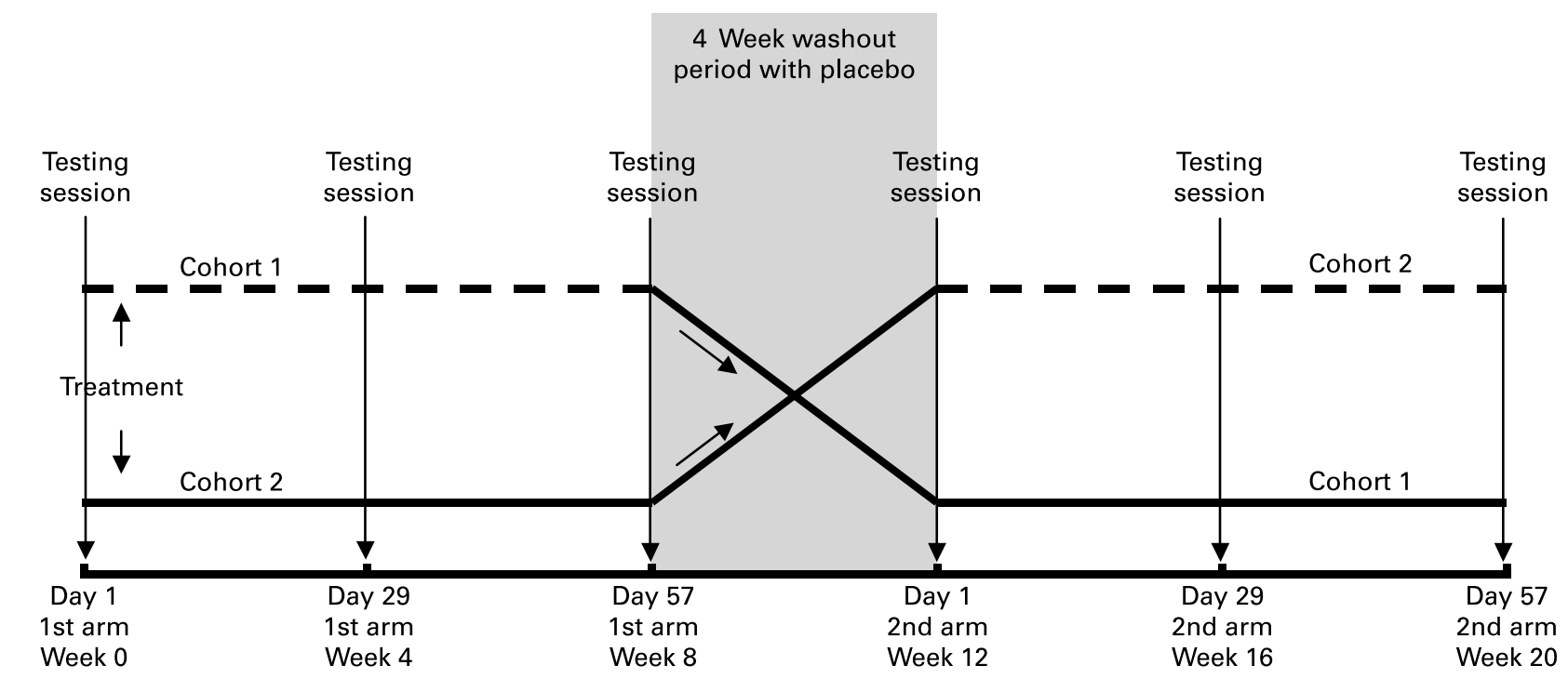

Fig. 1. Timelines of the study. Participants received either placebo (--) or $200 \mathrm{mg}$ ginseng (-) during the first arm of the study (weeks $0-8)$, and the opposite treatment in the second arm (weeks 12-20). All participants received placebo during the washout period (beginning of week 9 to end of week 12). Testing days took place on days 1,29 and 57 of each arm. 
(pre, post)) to reveal any acute, chronic and superimposed (i.e. acute upon chronic) effect.

\section{Results}

\section{Glycated $\mathrm{Hb}$}

There was no significant main effect of treatment or interaction with treatment revealed in either study 1 or study 2 (see Table 1).

\section{Fasting plasma insulin}

There was no significant main effect of treatment or interaction with treatment revealed in either study 1 or study 2 (see Table 1).

\section{Capillary finger prick blood glucose}

There was no significant main effect of treatment or significant interaction with treatment revealed in either study 1 or study 2 (see Table 2).

\section{Discussion}

To our knowledge, the present study is the first to assess the effect of chronic ingestion of Panax ginseng on indices of glucose regulation in healthy volunteers. The results show that $200 \mathrm{mg}$ Panax ginseng from two different sources, ingested daily for 4 and 8 weeks, has no effect on indices of glucose regulation (HbA1c, fasting plasma insulin, fasting plasma glucose). The results also suggest that there is no effect at the acute $3 \mathrm{~h}$ measurement point that follows a light breakfast, measured $180 \mathrm{~min}$ after ginseng ingestion, in healthy volunteers.

To date there have been a number of studies that have investigated the glycaemic effects, following chronic ingestion, of different members of the genus Panax family in a diabetic population. However, only three studies ${ }^{(8,10,12)}$ give sufficient detail, relating to the extract and dose used, to allow discussion. Two of the three studies have utilised Panax ginseng. The results of the present study are at odds with both of these previous studies. For example, in the first, Tetsutani et al. ${ }^{(8)}$ reported significant reductions in HbA1c following 3-4.5 g Panax ginseng administered for 24 months. In the second Vuksan et al. ${ }^{(12)}$ reported significant improvements in nine of the eleven indices of glucose regulation measured, following 12 weeks of Panax ginseng supplementation $(6 \mathrm{~g} / \mathrm{d})$. Interestingly, the two indices that Vuksan failed to find improvements on (i.e. HbA1c and fasting blood glucose levels) are two of the four indices of glucose regulation measured in the present study (however, it should be noted that both previous studies utilised diabetic populations, therefore direct comparisons between the present study, utilising non-diabetics, and these previous studies are problematic). In the third study, Vuksan et al. ${ }^{(10)}$ reported the effect following 8-week administration of Panax quinquefolius $(1 \mathrm{~g} / \mathrm{d})$ and reported significant reductions in $\mathrm{HbA} 1 \mathrm{c}$ and fasting blood glucose levels (again comparisons are problematic as differences between ginseng type and the population studied may explain the different profile of results). Given the caution advised earlier, the most parsimonious explanation for the discrepancies between the present study and those reported in a diabetic population would be differences in study duration, the extract under investigation and differences in the population studied. Further research is needed to investigate these effects in a non-diabetic population. Additionally, caution is advised when interpreting the present results as there are a number of limitations; the present study had reduced sample size as a result of missing data; the storage of the blood samples (i.e. $-20^{\circ} \mathrm{C}$ ) is not recommended as the stability of the sample is questionable and finally there is an imbalance of the number of males and females in study 2 .

The present study also allowed further consideration of the acute glycaemic effects of Panax ginseng ingestion that followed a light breakfast. The results revealed no difference between treatment and placebo, measured $180 \mathrm{~min}$ after ginseng ingestion. The rationale for this measurement point stems from an interest to investigate if the hypoglycaemic effects (previously reported) would persist beyond the clinically relevant $2 \mathrm{~h}$ measurement point. This is of interest as no study to date has investigated the glycaemic effects beyond this $2 \mathrm{~h}$ point. It is possible that ginseng continues to lower blood glucose levels past normoglycaemic levels

Table 1. Glycated $\mathrm{Hb}(\mathrm{HbA} 1 \mathrm{c})$ and fasting plasma insulin levels following 29 and $57 \mathrm{~d}$ of consecutive ginseng ingestion $(200 \mathrm{mg} / \mathrm{d})$ for two different ginseng extracts in healthy volunteers*

(Mean values with their standard errors)

\begin{tabular}{|c|c|c|c|c|c|c|c|}
\hline & \multirow[b]{2}{*}{ Treatment } & \multicolumn{2}{|c|}{ Day 1} & \multicolumn{2}{|c|}{ Day 29} & \multicolumn{2}{|c|}{ Day 57} \\
\hline & & Mean & SE & Mean & SE & Mean & SE \\
\hline \multicolumn{8}{|l|}{$\mathrm{HbA} 1 \mathrm{c}(\%$ of total $)$} \\
\hline \multirow[t]{2}{*}{ Study 1 ( $n$ 18) } & Placebo & 5.45 & 0.08 & $5 \cdot 32$ & 0.08 & 5.24 & 0.08 \\
\hline & $200 \mathrm{mg}$ & $5 \cdot 36$ & 0.07 & $5 \cdot 38$ & 0.09 & $5 \cdot 32$ & 0.09 \\
\hline \multirow[t]{2}{*}{ Study 2 ( $n 11)$} & Placebo & 5.45 & 0.11 & $5 \cdot 24$ & 0.09 & $5 \cdot 21$ & 0.08 \\
\hline & $200 \mathrm{mg}$ & 5.49 & 0.11 & 5.41 & 0.12 & $5 \cdot 23$ & 0.10 \\
\hline \multicolumn{8}{|c|}{ Plasma insulin $(\mu \mathrm{lU} / \mathrm{ml})$} \\
\hline \multirow[t]{2}{*}{ Study 1 ( $n$ 17) } & Placebo & $10 \cdot 51$ & 0.98 & $13 \cdot 85$ & 1.36 & 13.66 & 0.94 \\
\hline & $200 \mathrm{mg}$ & 11.03 & 0.73 & 12.93 & 2.04 & 14.13 & 2.90 \\
\hline \multirow[t]{2}{*}{ Study 2 ( $n$ 12) } & Placebo & 13.99 & 1.53 & $13 \cdot 12$ & 0.94 & 13.57 & 1.63 \\
\hline & $200 \mathrm{mg}$ & 13.98 & 1.16 & 14.57 & 1.09 & $13 \cdot 12$ & 0.81 \\
\hline
\end{tabular}

* For details of subjects and procedures, see Participants and methods. Study 1 used G115 ginseng and study 2 used Cheong Kwan Jang ginseng. 
Table 2. Blood glucose levels (mmol/l) following 29 and $57 \mathrm{~d}$ of consecutive ginseng ingestion $(200 \mathrm{mg} / \mathrm{d})$ are represented for two different ginseng extracts, in healthy volunteers*

(Mean values with their standard errors)

\begin{tabular}{|c|c|c|c|c|c|c|c|c|c|c|c|c|c|}
\hline & \multirow[b]{3}{*}{ Treatment } & \multicolumn{4}{|c|}{ Day 1} & \multicolumn{4}{|c|}{ Day 29} & \multicolumn{4}{|c|}{ Day 57} \\
\hline & & \multicolumn{2}{|c|}{ Pre-dose } & \multicolumn{2}{|c|}{$3 \mathrm{~h}$ post-dose } & \multicolumn{2}{|c|}{ Pre-dose } & \multicolumn{2}{|c|}{$3 \mathrm{~h}$ post-dose } & \multicolumn{2}{|c|}{ Pre-dose } & \multicolumn{2}{|c|}{$3 \mathrm{~h}$ post-dose } \\
\hline & & Mean & SE & Mean & SE & Mean & SE & Mean & SE & Mean & SE & Mean & SE \\
\hline \multirow[t]{2}{*}{ Study 1 (n 23) } & Placebo & 4.90 & 0.19 & 4.94 & 0.14 & 5.05 & 0.11 & $5 \cdot 15$ & 0.16 & $5 \cdot 00$ & 0.16 & $5 \cdot 42$ & 0.11 \\
\hline & $200 \mathrm{mg}$ & 5.04 & 0.16 & 5.07 & 0.15 & $5 \cdot 18$ & 0.15 & $5 \cdot 22$ & 0.20 & 5.29 & 0.10 & $5 \cdot 21$ & 0.16 \\
\hline \multirow[t]{2}{*}{ Study 2 ( $n$ 14) } & Placebo & 5.65 & 0.14 & 5.49 & 0.19 & 5.39 & 0.17 & $5 \cdot 16$ & 0.19 & $5 \cdot 36$ & 0.17 & $5 \cdot 30$ & 0.17 \\
\hline & $200 \mathrm{mg}$ & 5.43 & 0.16 & $5 \cdot 26$ & 0.23 & 5.53 & 0.14 & $5 \cdot 11$ & 0.34 & 5.55 & 0.19 & 5.41 & 0.23 \\
\hline
\end{tabular}

* For details of subjects and procedures, see Participants and methods. Study 1 used G115 ginseng and study 2 used Cheong Kwan Jang ginseng. Pre-dose values represent blood glucose levels following an overnight fast and measured prior to the ingestion of that day's ginseng dose. Post-dose values represent blood glucose levels measured $3 \mathrm{~h}$ post-dose. A light breakfast was consumed immediately following ginseng dose on each study day.

(normoglycaemic levels are typically reached by $120 \mathrm{~min}$ ). As the present study is the first study to measure the acute glucoregulatory effects of ginseng beyond this $2 \mathrm{~h}$ point it is not valid to compare results of the present study to those that have measured the glycaemic effect during an oral glucose tolerance test (incorporating the $2 \mathrm{~h}$ measurement point) and the fact that participants' blood glucose levels will have returned to normoglycaemic levels. Further research is needed.

The present results may give insight into the safety of Panax ginseng use in healthy non-diabetic populations. Despite there being no health risk associated with the acute postprandial hypoglycaemic properties of ginseng (these effects being considered advantageous in diabetic populations), there may be some concerns relating to the duration of these effects and further concerns when such products are ingested in a fasted state. One might assume that the actions of glucagon would counteract any further hypoglycaemic effect, once glucose levels have returned to normal levels following a challenge, at later time-points. However, such a control mechanism did not appear in a group of healthy volunteers whose fasted blood glucose level dropped by as much as $20 \%$, when measured 120 min after Panax ginseng ingestion (no later time-points were measured $)^{(13)}$. Therefore, the present results suggest that ginseng's acute hypoglycaemic effect does not continue once glucose levels have normalised, after consuming a light breakfast (obviously, we cannot comment if ginseng lowered blood glucose levels at earlier timepoints and we cannot comment if this effect would be the same, if participants were fasted overnight). This is of particular interest as Panax ginseng is by far the most commonly consumed member of the genus, not only for its purported health benefits but also for its effect on human behaviour ${ }^{(20)}$.

The mechanisms underlying ginseng's glycaemic effects are not clear at present. However, animal and human data would suggest three possible mechanisms that could potentially account for the modulation in blood glucose levels: (1) modulation of glucose uptake; (2) modulation of glucose disposal; (3) modulation of insulin secretion. For example, Lai et al. ${ }^{(21)}$ showed ginsenoside $\mathrm{Rh}_{2}$ led to reductions in plasma glucose levels and an overexpression of the GLUT4 glucose transporter in rats. Wang et al. ${ }^{(22)}$ provided evidence that ginseng increases aerobic glycolysis through enhancing the activities of citrate synthetase, malate dehydrogenase and succinate dehydrogenase, cytochrome oxidase (four rate-limiting enzymes in aerobic glycolysis). Chung et al. ${ }^{\text {(23) }}$ reported that $50 \mathrm{mg} / \mathrm{kg}$ aqueous Panax ginseng root body and rootlets administered for $28 \mathrm{~d}$ decreased the activity of the rate-limiting gluconeogenic enzyme glucose-6-phosphatase in liver preparations in diabetic mice by 46 and $20 \%$. Liu et al. ${ }^{(24)}$ provided evidence that the oral ingestion of Panax ginseng improved insulin sensitivity that resulted in the modulation (i.e. lowering) of circulating glucose levels.

In conclusion, the present study suggests that the chronic ingestion of Panax ginseng has no effect on indices of glucose regulation in a non-diabetic sample. Additionally, there was no difference in glucose levels when measured $180 \mathrm{~min}$ post-dose. Further research is needed.

\section{Acknowledgements}

Funding for the completion of study 1 was provided by Pharmaton SA, Lugano, Switzerland; and for study 2 by Korea Ginseng Corporation, Seoul, Korea. There are no conflicts of interest. J. L. R., A. B. S., A. M. and D. O. K. contributed to the design, data collection and data analysis and contributed to the writing of this report. J. F. advised on HbA1c and carried out the HbA1c analysis.

\section{References}

1. Mokdad AH, Ford ES, Bowman BA, et al. (2003) Prevalence of obesity, diabetes, and obesity-related health risk factors, 2001. JAMA 289, 76-79.

2. Turner RC, Cull CA, Frighi V, et al. (2000) Glycemic control with diet, sulfonylurea, metformin, or insulin in patients with type 2 diabetes mellitus: progressive requirement for multiple therapies (UKPDS 49), UK Prospective Diabetes Study (UKPDS) group. JAMA 281, 2005-2012.

3. Roglic G, Unwin N, Bennett PH, et al. (2005) The burden of mortality attributable to diabetes. Diabetes Care $\mathbf{2 8}$, $2130-2135$.

4. Xie JT, Zhou YP, Dey L, et al. (2002) Ginseng berry reduces blood glucose and body weight in $\mathrm{db} / \mathrm{db}$ mice. Phytomedicine 9, 254-258.

5. Ohnishi Y, Takagi S, Miura T, et al. (1996) Effects of ginseng radix on GLUT2 protein content in mouse liver in normal and epinephrine induced hypoglycaemic mice. Biol Pharm Bull 19, $1238-1240$. 
6. Xie JT, Wang CZ, Wang AB, et al. (2005) Antihyperglycemic effects of total ginsenosides from leaves and stem of panax ginseng. Acta Pharmacol Sin 26, 1104-1110.

7. Sotaniemi EA, Haapakoski E \& Rautio A (1995) Ginseng therapy in non-insulin diabetic patients. Diabetes Care 18, 1373-1375.

8. Tetsutani T, Yamamura M, Yamaguchi T, et al. (2000) Can red ginseng control blood glucose in diabetic patients. Ginseng Rev 28, 44-47.

9. Vuksan V, Sievenpiper JL, Koo VYY, et al. (2000) American ginseng (Panax quinquefolius L) reduces postprandial glycemia in nondiabetic subjects and subjects with type 2 Diabetes Mellitus. Arch Int Med 160, 1009-1013.

10. Vuksan V, Xu Z \& Jenkins AL (2000) American ginseng improves long term glycaemic control in type two diabetes: double blind placebo controlled crossover trial. Diabetes $\mathbf{4 9}$, Suppl. 1, A95, (abstract).

11. Vuksan V, Sievenpiper JL, Wong J, et al. (2001) American ginseng (Panax quinquefolius L.) attenuates postprandial glycemia in a time-dependent but not dose-dependent manner in healthy individuals. Am J Clin Nutr 73, 753-758.

12. Vuksan V, Sung MK, Sievenpiper JL, et al. (2006) Korean red ginseng (panax ginseng) improves glucose and insulin regulation in well controlled type 2 diabetes: results of a randomized, double-blind, placebo-controlled study of efficacy and safety. Nutr Metab Cardiovasc Dis 18, 46-56.

13. Reay JL, Kennedy DO \& Scholey AB (2005) Single doses of panax ginseng (G115) reduce blood glucose levels and improve cognitive performance during sustained mental activity. J Psychopharmacol 19, 357-365.

14. Reay JL, Kennedy DO \& Scholey AB (2006) The glycaemic effect of single doses of panax ginseng (G115) in young healthy volunteers. Br J Nutr 96, 639-642.

15. Dascalu A, Sievenpiper JL, Jenkins AL, et al. (2007) Five batches representative of Ontario-grown American ginseng root produce comparable reductions of postprandial gycemia in healthy individuals. Can J Physiol Pharmacol 85, 856-864.

16. Sievenpiper JL, Arnason JT, Leiter LA, et al. (2002) Variable effects of American ginseng: a batch of American ginseng (Panax quinquefolius L.) with a depressed ginsenoside profile does not affect postprandial glycemia. Eur J Clin Nutr 57, 243-248.

17. Sievenpiper JL, Arnason JT, Leiter LA, et al. (2003) Possible opposing effects of Asian ginseng (Panax ginseng C.A. Meyer) on glycemia: results of two acute dose escalation studies. J Am Coll Nutr 22, 524-532.

18. Sotaniemi EA, Haapakoski E \& Rautio A (1995) Ginseng therapy in non-insulin diabetic patients. Diabetes Care 18, $1373-1375$

19. Price CP \& Koller PU (1988) A multicentre study of the new Reflotron system for the measurement of urea, glucose, triacylglycerols, cholesterol, gamma-glutamyltransferase and haemoglobin. J Clin Chem Clin Biochem 26, 233-250.

20. Kennedy DO \& Scholey AB (2003) Ginseng: potential for the enhancement of cognitive performance and mood. Pharmacol Biochem Behav 75, 687-700.

21. Lai DM, Tu YK, Liu IM, et al. (2006) Mediation of beta-endorphin by ginsenoside Rh2 to lower plasma glucose in streptozotocin-induced diabetic rats. Planta Med 72, 9-13.

22. Wang LC, Wang B, Ng SY, et al. (2006) Effects of ginseng saponins on beta-amyloid-induced amnesia in rats. J Ethnopharmacol 103, 103-108.

23. Chung SH, Choi CG \& Park SH (2001) Comparisons between white ginseng radix an rootlet for antidiabetic activity and mechanism in KKAy mice. Arch Pharm Res 24, 214-218.

24. Liu TP, Liu IM \& Cheng JT (2005) Improvement of insulin resistance by panax ginseng in fructose-rich chow-fed rats. Horm Metab Res 37, 146-151. 